\title{
Analysis and three-dimensional visualization of collagen in artificial scaffolds using nonlinear microscopy techniques
}

\section{Eva Filová}

Institute of Experimental Medicine

Academy of Sciences of the Czech Republic

Vídeňská 1083

14220 Prague, Czech Republic

\section{Zuzana Burdíková}

Institute of Physiology

Academy of Sciences of the Czech Republic

Department of Biomathematics

Vídeňská 1083

14220 Prague, Czech Republic

\section{Michala Rampichová}

Institute of Experimental Medicine

Academy of Sciences of the Czech Republic

Vídeňská 1083

14220 Prague, Czech Republic

and

Charles University in Prague

Second Faculty of Medicine

Institute of Biophysics

$\checkmark$ Úvalu 84

15006 Praha 5, Czech Republic

\section{Paolo Bianchini}

Italian Institute of Technology

Nanophysics

via Morego 30

I16163 Genova, Italy

\section{Martin Čapek}

Institute of Physiology

Academy of Sciences of the Czech Republic

Department of Biomathematics

Vídeňská 1083

14220 Prague, Czech Republic

$$
\text { and }
$$

Czech Technical University in Prague

Faculty of Biomedical Engineering

Sítná 3105

27201, Kladno, Czech Republic

\section{Eva Košt'áková}

Technical University of Liberec

Faculty of Textile Engineering

Studentská 2

46117 Liberec, Czech Republic

\section{Evzen Amler}

Institute of Experimental Medicine

Academy of Sciences of the Czech Republic

Vídeňská 1083

14220 Prague, Czech Republic and

Charles University in Prague

Second Faculty of Medicine

Institute of Biophysics

V Úvalu 84

15006 Praha 5, Czech Republic

\author{
Lucie Kubínová \\ Institute of Physiology \\ Academy of Sciences of the Czech Republic \\ Department of Biomathematics \\ Vídeňská 1083 \\ 14220 Prague, Czech Republic
}

\begin{abstract}
Extracellularly distributed collagen and chondrocytes seeded in gelatine and poly- $\varepsilon$-caprolactone scaffolds are visualized by two-photon excitation microscopy (TPEM) and second-harmonic generation (SHG) imaging in both forward and backward nondescanned modes. Joint application of TPEM and SHG imaging in combination with stereological measurements of collagen enables us not only to take high-resolution 3-D images, but also to quantitatively analyze the collagen volume and a spatial arrangement of cell-collagen-scaffold systems, which was previously impossible. This novel approach represents a powerful tool for the analysis of collagen-containing scaffolds with applications in cartilage tissue engineering. (ㅇ 2010 Society of Photo-Optical Instrumentation Engineers. [DOI: 10.1117/1.3509112]
\end{abstract}

Keywords: second-harmonic generation imaging; multiphoton microscopy; tissue engineering; artificial scaffolds; collagen.

Paper 10041RR received Jan. 27, 2010; revised manuscript received Sep. 16, 2010; accepted for publication Sep. 21, 2010; published online Dec. 23, 2010.

\section{Introduction}

Multiphoton microscopy is an evolving high-resolution technique that has been successfully applied in many fields of biomedicine. ${ }^{1-3}$ Nonlinear microscopy can employ two different types of signals, fluorescence and second-harmonic generation (SHG), to image biological structures with subcellular resolution. Two-photon excited fluorescence imaging is a powerful technique for monitoring the dynamic behavior of chemical components of tissues, whereas SHG imaging provides novel ways to study their spatial distribution. ${ }^{4}$ Two-photon excitation (TPE) of fluorescent molecules is a process that involves the simultaneous absorption of two photons whose total energy equals the energy for producing a molecular transition to an excited state, while the SHG is a coherent nonlinear process that takes place in certain mesoscopic structures lacking centrosymmetry-such as collagen, myosin, and microtubules-when excited by an extremely intense light. ${ }^{5}$ To achieve the required photon density while maintaining an average laser power at a reasonable level, the laser light is concentrated into short pulses (about $200 \mathrm{fs}$ ) with a repetition rate of about $80 \mathrm{MHz}$. The wavelength of the emitted light is exactly one half of the excitation wavelength, and the emission intensity is proportional to the square of the excitation radiation intensity in the focal point. ${ }^{6}$ The advantages of SHG imaging include

1083-3668/2010/15(6)/066011/7/\$25.00 (C) 2010 SPIE
Address all correspondence to: Lucie Kubínová, Institute of Physiology, Academy of Sciences of the Czech Republic, v.v.i., Tel: +420 296442 314; Fax: +420 241062 488; E-mail: kubinova@biomed.cas.cz. 
high sensitivity and resolution, absence of photobleaching, lack of autofluorescence, and non-destructive imaging of native tissues.

SHG imaging has been used for the identification of type I collagen in fascia; ${ }^{7}$ tendon; ${ }^{8,9}$ skin $;{ }^{5,10}$ and liver, where it was also used as a marker of liver cirrhosis. ${ }^{11}$ Structural details of different types of collagen as well as its morphological changes observed during aging or disease were also studied by SHG imaging. ${ }^{12}$

The major components of extracellular matrix in hyaline cartilage include type II collagen $(\approx 60 \%$ of the dry weight), hyaluronic acid, and proteoglycans. ${ }^{13}$ Cartilage damage as a consequence of diseases such as osteoarthritis is characterized by a decreased concentration of aggrecan, a shorter length of glycosaminoglycan chains, increased water content, and collagen degradation or altered organization. These changes result in an increase of tissue permeability and reduced cartilage stiffness. ${ }^{14,15}$

The aim of regenerative medicine is renovation or reconstruction of missing tissues; thus, it is largely dependent on progress in tissue engineering. Innovative scaffolds as well as application of novel visualization methods are hence extensively studied in many laboratories. Implantation of biodegradable scaffolds seeded with chondrocytes is an example of a promising technique used in modern therapy of osteochondral defects. The quality of implanted cell-seeded scaffolds, which is characterized by differentiated cells as well as extracellular matrix production, is an important factor for cartilage regeneration. ${ }^{16,17}$ Therefore, highly sensitive rapid visualization of collagen and cells is highly demanded to assess the quality of prepared implants.

Different scaffolds are characterized by differences in autofluorescence, TPE microscopy (TPEM), or SHG signals. ${ }^{18}$ Both confocal laser-scanning microscopy (CLSM) and TPEM enable visualization of collagen using immunohistochemical staining. However, the fibrous or homogenous character of the collagen cannot be observed, since the antibodies are bound to the epitopes on the surface. On the other hand, SHG visualizes the whole collagen fibers even in the deeper part of the scaffold, which enables a spatial characterization with high sensitivity of the collagen in scaffolds of high thickness. The visualization of cells on the scaffold as well as extracellular matrix formation makes it possible to assess the proliferation and differentiation state of cells as well as collagen production and is of prime importance in tissue engineering. Simultaneous application of CLSM, TPEM, and SHG yielded imaging in great detail. The aim of the presented study was to test the sensitivity of nonlinear microscopic techniques for visualization of cells together with collagen and to quantitatively analyze collagen distribution.

\section{Methods}

\subsection{Sample Preparation and Seeding}

The scaffolds were produced from gelatine (Gelita Imagel BT, Type 68 917, gel 270 g Bloom, Gelita AG, Germany). A gelatine scaffold was prepared using $5 \%(\mathrm{w} / \mathrm{w})$ gelatine solution in distilled water at $40^{\circ} \mathrm{C}$. The solution was thoroughly stirred in an agitator; subsequently, the foam was dried at room temperature for $48 \mathrm{~h}$. Samples were then crosslinked using $0.7 \%(\mathrm{w} / \mathrm{w}) \mathrm{N}$-(3-dimethylaminopropyl)- $\mathrm{N}^{\prime}$-ethylcarbodiimide hydrochlorid (type 03450, Sigma-Aldrich) in acetone/water (8:2 ratio) for $24 \mathrm{~h}$ at room temperature, and dried at room temperature for $48 \mathrm{~h}$. The samples were then washed with distilled water to remove unwanted chemicals.

A poly- $\varepsilon$-caprolactone (PCL) foam scaffolds were prepared by salt-leaching techniques using 7\% (w/w) PCL (Mw $80 \times 10^{3}$, Sigma-Aldrich) dissolved in ethanol/chloroform mixture (1:9). $\mathrm{NaCl}$ particles were mixed with the solution mechanically. After solvent evaporation, salt particles were removed by stirring in distilled water.

\subsection{Chondrocyte Isolation and Culture}

Small pieces of cartilage were taken from the left femoral trochlea of a rabbit under general anesthesia. After a 14-h digestion period in $1 \mathrm{mg} / \mathrm{mL}$ collagenase solution (collagenase crude $816 \mathrm{PZS} / \mathrm{g}$, Sevapharma), chondrocytes were collected and cultured for 14 days in Iscove's modified Dulbecco's medium (IMDM) supplemented with $10 \%$ foetal bovine serum (FBS), penicillin/streptomycin (100 IU/mL and $100 \mu \mathrm{g} / \mathrm{mL}$, respectively), $400 \mathrm{mM}$ L-glutamine, $100 \mathrm{nM}$ dexamethasone, $40 \mu \mathrm{g} / \mathrm{mL}$ ascorbic acid-2-phosphate, and ITS-X $(10 \mu \mathrm{g} / \mathrm{mL}$ insulin, $5.5 \mathrm{mg} / \mathrm{L}$ transferrin, $6.7 \mu \mathrm{g} / \mathrm{L}$ sodium selenite, and $2 \mathrm{mg} / \mathrm{L}$ ethanolamine). Chondrocytes were seeded on 5\% gelatine foams and PCL foams at a density of $8.0 \times 10^{5}$ and $3.0 \times 10^{5}$ cells $/$ scaffold, respectively (scaffold diameter $=6 \mathrm{~mm}$, height $=1 \mathrm{~mm}$ ). The cells were incubated at $37^{\circ} \mathrm{C}$ in a $5 \% \mathrm{CO}_{2}$ atmosphere for 14 days.

\subsection{Sample Staining}

Scaffolds or cartilage samples were fixed by ethanol $\left(-20^{\circ} \mathrm{C}\right)$ and stained by propidium iodide [5 $\mu \mathrm{g} / \mathrm{mL}$ in phosphate-buffered saline (PBS)]. Indirect immunofluorescence staining was used to detect type II collagen. Samples were fixed with $10 \%$ formaldehyde in PBS for $10 \mathrm{~min}$, washed in PBS, and then incubated in $3 \%$ FBS in PBS $/ 0.1 \%$ Triton X-100 at room temperature for $30 \mathrm{~min}$.

The samples were incubated with the primary antibody against type II collagen, clone II-II6B3, obtained from Developmental Studies Hybridoma Bank developed under the auspices of the National Institute of Child Health and Human Development (NICHD) and maintained by the University of Iowa, Department of Biological Sciences (Iowa City, IA 52242) (dilution 1:20; 1-h incubation at room temperature). After three washes with PBS/0.05\% Tween 20 after 3, 10, and $15 \mathrm{~min}$, the samples were incubated with a secondary antibody Cy3-conjugated Donkey Anti-Mouse IgG $(\mathrm{H}+\mathrm{L})$ (dilution 1:300; 45-min incubation at room temperature, light protected). Samples were again washed with PBS/ $0.05 \%$ Tween 20 and once with PBS; subsequently, antifading solution was added $[\mathrm{PBS} / 90 \%$ glycerol $/ 2.5 \%$ 1,4-diazabicyclo(2,2,2)octane (DABCO)]

\subsection{Image Acquisition}

All images were acquired by a Leica TCS SP2 acousto-optical beamsplitter (AOBS) multiphoton confocal laser scanning 


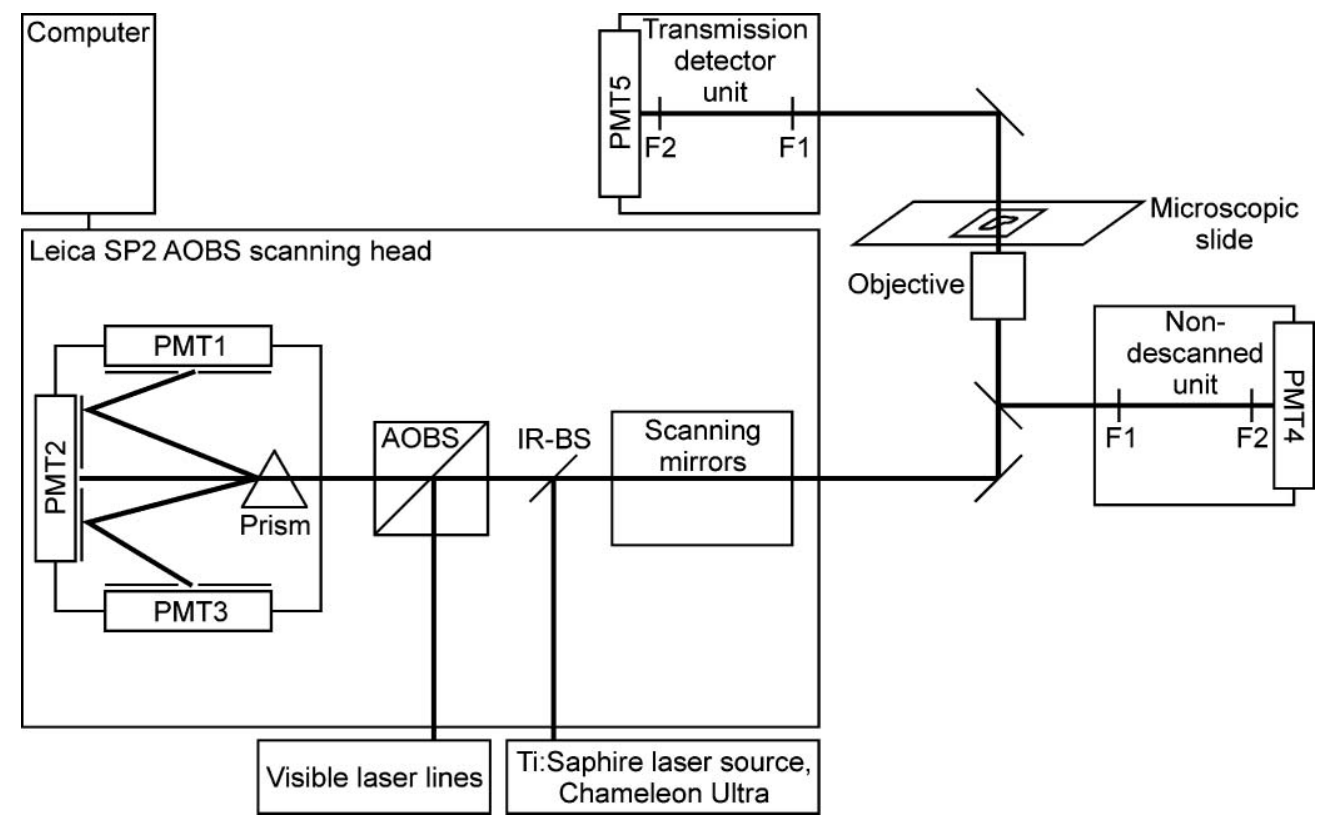

Fig. 1 Diagrammatic layout of the experimental setup based on a confocal laser scanning microscope Leica SP2 AOBS equipped with one- and two-photon lasers. PMT1, PMT2, and PMT3, photomultiplier tubes in the scanning head for descanned detection of one- and two-photon imaging of fluorescence or autofluorescence signal; PMT4, photomultiplier for nondescanned detection of SHG signal in the backward mode; PMT5, transmission detector for nondescanned detection of SHG signal in the forward mode; F1, E700SP short-pass filter; F2, $430 D F 15$ bandpass filter; IR-BS, infrared beamsplitter.

microscope based on Leica DM IRE2 inverted microscope and equipped with the following light sources: Ar laser $(458 \mathrm{~nm} / 5$ $\mathrm{mW}, 476 \mathrm{~nm} / 5 \mathrm{~mW}, 488 \mathrm{~nm} / 20 \mathrm{~mW}, 514 \mathrm{~nm} / 20 \mathrm{~mW}), \mathrm{HeNe}$ lasers (543 nm/1.2 mW, $633 \mathrm{~nm} / 10 \mathrm{~mW})$ for one-photon excitation, and a mode-locked Ti:Sapphire Chameleon Ultra laser (Coherent Inc., Santa Clara, California), tuneable from 690 to $1040 \mathrm{~nm}$ for TPE (Fig. 1).

\subsubsection{Imaging of fluorescence signal using one-photon excitation}

Images of structures stained by propidium iodide were acquired using one-photon excitation $\left(\lambda_{\mathrm{exc}}=543 \mathrm{~nm}\right.$ and $\lambda_{\mathrm{em}}=610$ to $645 \mathrm{~nm}$ ) via a photomultiplier located in the scanning head (Fig. 1). Type II collagen stained by $\mathrm{Cy} 3$ was examined at $\lambda_{\mathrm{exc}}$ $=514 \mathrm{~nm}$ and $\lambda_{\mathrm{em}}=555$ to $600 \mathrm{~nm}$, also using an internal detector. The gelatine and PCL scaffold thicknesses were 214 \pm 75 and $195 \pm 37 \mu \mathrm{m}$, respectively [mean and standard deviation (SD)].

\subsubsection{SHG imaging}

In our setup shown in Fig. 1, the SHG signal was detected in three ways: (1) the backward descanned mode when the signal passed through the scanning head of the confocal microscope to PMT1, (2) the backward nondescanned mode when the signal was collected directly behind the objective by PMT4, and (3) the forward nondescanned mode when the signal passed through the sample into PMT5. For collagen detection the laser was tuned at the wavelength of $860 \mathrm{~nm}$, hence the SHG signal was expected at $430 \mathrm{~nm}$. In the descanned mode, the SHG signal was detected in the range of 420 to $440 \mathrm{~nm}$. In a nondescanned configuration, which is supposed to improve the signal- to-noise ratio in the acquired images, the external detector was placed behind a 700-nm short-pass filter, which attenuates IR light, followed by a 430-nm bandpass filter with a bandwidth of $15 \mathrm{~nm}$. To confirm the detection of the SHG signal we ensured that the signal disappeared when the excitation wavelength was changed to $800 \mathrm{~nm}$, then reappeared after setting up a bandpass filter to $400 \mathrm{~nm}$.

\subsubsection{Imaging fluorescence signal using TPE}

The fluorescence of the stained cells and/or autofluorescence of the scaffolds was also detected by TPEM using the internal detectors (in the descanned mode, see Fig. 1). An excitation wavelength of $860 \mathrm{~nm}$ was used to enable a simultaneous acquisition of the SHG signal. For detection, different wavelength ranges were applied using lambda scans to get as strong as possible autofluorescence of the samples.

\subsection{Three-Dimensional Reconstructions}

Further information on the spatial arrangement of tissues was obtained from three-dimensional (3-D) reconstructions based on series of optical sections of the specimen. ${ }^{19}$ The 3 -D visualization utilized a maximum intensity projection of these series using Leica LCS software and, further, volume rendering by the Volume plug-in module developed in Ellipse3D software environment (ViDiTo, Košice, Slovakia).

\section{Results}

SHG imaging was used for the identification of type II collagen in an extracellular matrix. To observe and detect 


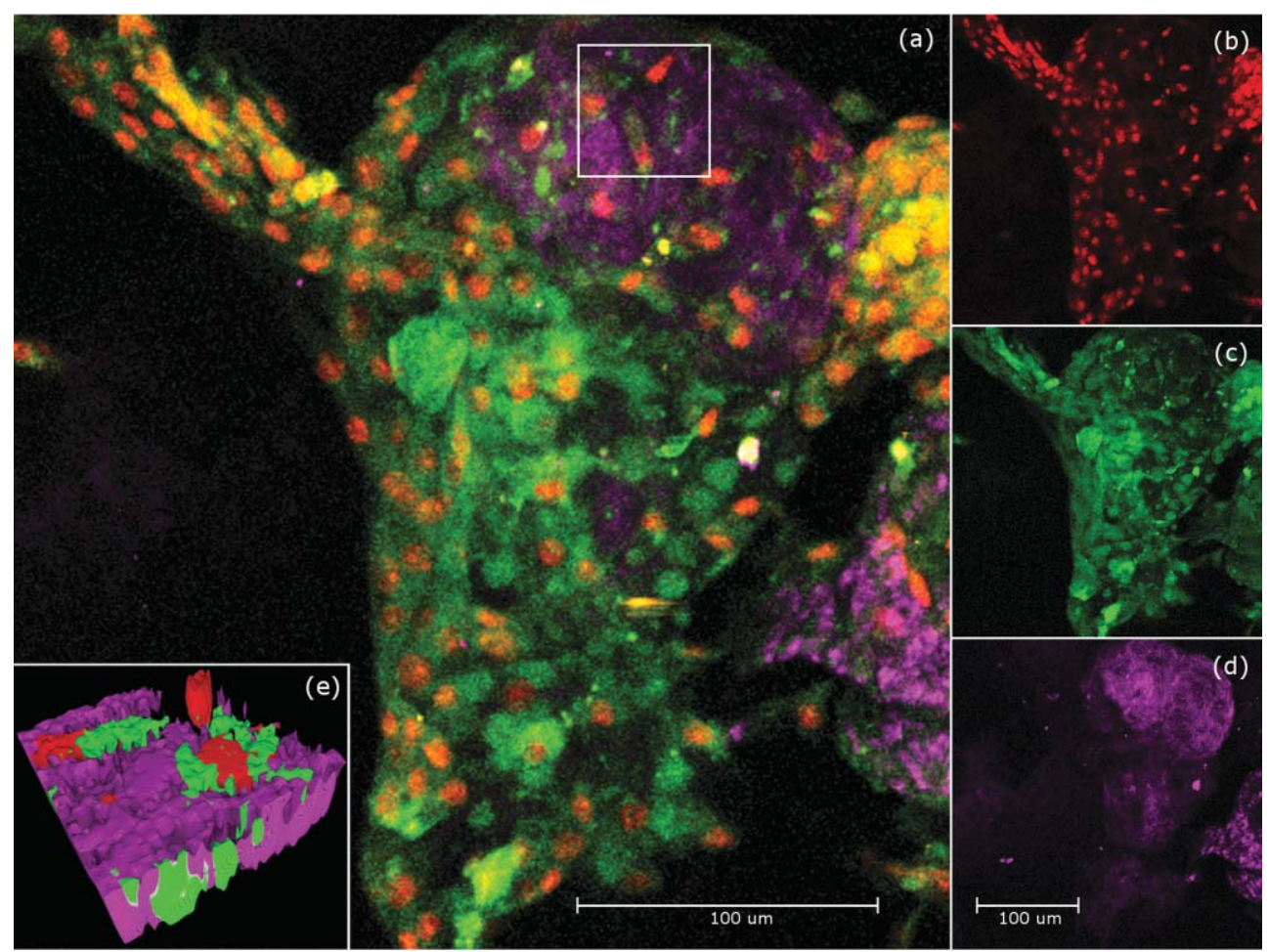

Fig. 2 Detection of collagen in PCL scaffold. (a) Maximum intensity projection of a 30- $\mu$ m-thick stack of images [overlay of (b), (c), and (d)], $20 \times$ objective, $2 \times$ zoom, scale $=100 \mu \mathrm{m}$. The white square marked region of interest with detected collagen of the size of $36 \times 36 \times 19 \mu \mathrm{m}^{3}$ is shown in more detail using a 3-D reconstruction (e). (b) Cell nuclei stained by propidium iodide (red) detected by TPE in the descanned mode; (c) procollagen labeled with Cy3 detected by TPE in a green channel; and (d) collagen detected by SHG imaging in the backward mode (magenta), autofluorescence of the scaffold is also detected.

collagen, chondrocytes were seeded on gelatine and PCL foams. The cell densities on $5 \%$ gelatine foam and the PCL scaffolds were $1.4 \times 10^{4}$ and $5.5 \times 10^{4}$ cells $/ \mathrm{mm}^{3}$, respectively. Chondrocytes adhered well to the surface of PCL [Fig. 2(a)] and gelatine scaffolds (Fig. 3). As expected, cells proliferated effectively on scaffolds and were approximately $80 \mu \mathrm{m}$ deep after 5 days. The cells were found to be grouped in large islands or formed continuous layers in pockets [Figs. 2(a), 2(b) and 3(a)].

Chondrocyte proliferation was linked to production of the extracellular matrix proteins. Collagen as a typical extracellular cartilage protein was visualized, first, by CLSM using fluorescently labeled monoclonal antibodies against type II collagen. Fluorescence staining clearly revealed only a sparse presence of collagen in gelatine foams, however, a high amount of type II collagen was detected in PCL scaffolds [Fig. 2(c)]. We performed stereological measurements of collagen volumes in five 100- $\mu \mathrm{m}$-deep series acquired from three PCL scaffolds. The measurements revealed $5.6 \pm 1.8 \%\left(7.49 \times 10^{5} \mu \mathrm{m}^{3}\right)$ and $2.6 \pm 2.3 \%\left(3.43 \times 10^{5} \mu \mathrm{m}^{3}\right)$ (mean and SD) of collagen volume related to volume of one series acquired from the scaffold $\left(140.57 \times 10^{5} \mu \mathrm{m}^{3}\right)$ using nondescanned and descanned modes, respectively.

Conventional confocal microscopy images were compared with the SHG imaging in both forward and backward nondescanned modes. Well detectable signals were observed in PCL and gelatine scaffolds (Figs. 2 and 3). Detection of collagen by SHG imaging corresponded well with collagen de- tection by immunohistochemical staining (Figs. 3 and 4). The autofluorescence signal was collected with the aim to visualize the scaffold structure. The collagen signal was detected at sites of high chondrocyte density on the scaffold.

Fluorescence collagen signal was acquired using CLSM [Fig. 4(c)]. An SHG signal of high intensity was detected in the extracellular matrix of cartilage as well as in the close vicinity of cells, indicating newly synthesized collagen. Beside collagen, chondrocytes and scaffolds were also visualized using a simultaneous acquisition of SHG and TPEM signals [Figs. 2(a), 3(b1) and 3(c1)].

The volume of visualized collagen (backward versus forward mode SHG signal) produced by cells in scaffolds was measured. Gray-scale-level thresholding of the 3-D image data was applied to identify regions of high-intensity SHG signal. Volumes of these regions were then calculated from the number of their voxels. Using threshold of 30 ( 0 to 255 gray scale), the volumes of collagen detected in backward and forward modes were $5.36 \times 10^{5} \mu \mathrm{m}^{3}$ and $1.54 \times 10^{5} \mu \mathrm{m}^{3}$, respectively. The volume of the whole series was $140.57 \times 10^{5} \mu \mathrm{m}^{3}$. This indicated a slightly higher sensitivity of the backward mode to collagen compared to the forward one.

A combination of TPEM signals (propidium iodide, scaffold autofluorescence) and SHG signal from collagen was used for 3-D reconstructions. These multimodal 3-D images simultaneously revealed both collagen and scaffold architecture, and cell localization [Figs. 2(e), 3(b2), 3(c2) and Videos 1, 2, and 3]. 

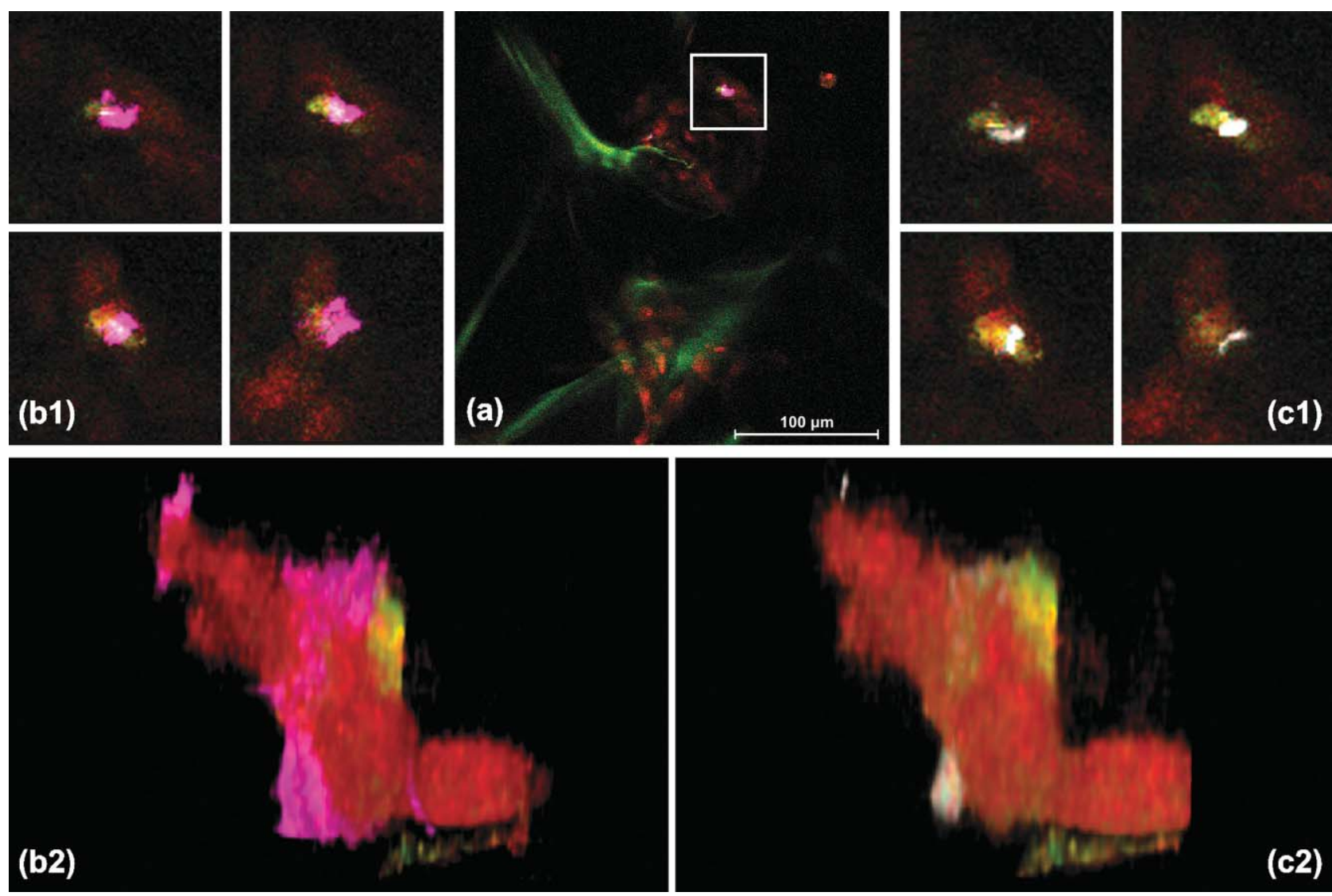

Fig. 3 Gelatine scaffold imaging by SHG in the backward and forward modes. (a) An optical section using TPE; cell nuclei stained by propidium iodide (red), scaffold autofluorescence (green), and SHG imaging in the backward mode (magenta), scale $=100 \mu \mathrm{m}$. The white-square-marked region of interest is shown in more detail in four optical sections of the image stack, $10 \mu \mathrm{m}$ apart: (b1) SHG imaging in the backward mode (magenta), (c1) SHG imaging in the forward mode (cyan). The 3-D image of the region of interest shown in (a) demonstrates a stronger SHG signal detected in the backward mode (b2) when compared to the forward mode (c2). The dimensions of the reconstructed volumes were $60 \times 60 \times 70 \mu \mathrm{m}^{3} ; 20 \times$ objective.

\section{Discussion}

SHG imaging was used for the analysis of various collagencontaining tissues, such as skin, tendon, muscles, cartilage, fascia, and endometrium. ${ }^{7-12}$ Structural changes of collagen in cryopreserved cartilage demonstrated by SHG imaging were also reported. ${ }^{12}$
Our SHG images of cartilage showed a homogeneous signal from collagen in the extracellular matrix [Figs. 4(a) and 4(b)]. This is in accordance with immunohistochemical staining of type II collagen [Fig. 4(c)].

The optimal technique of detecting SHG signal of collagen in scaffolds was investigated in the presented study, and both the backward and forward modes were tested. In general, SHG
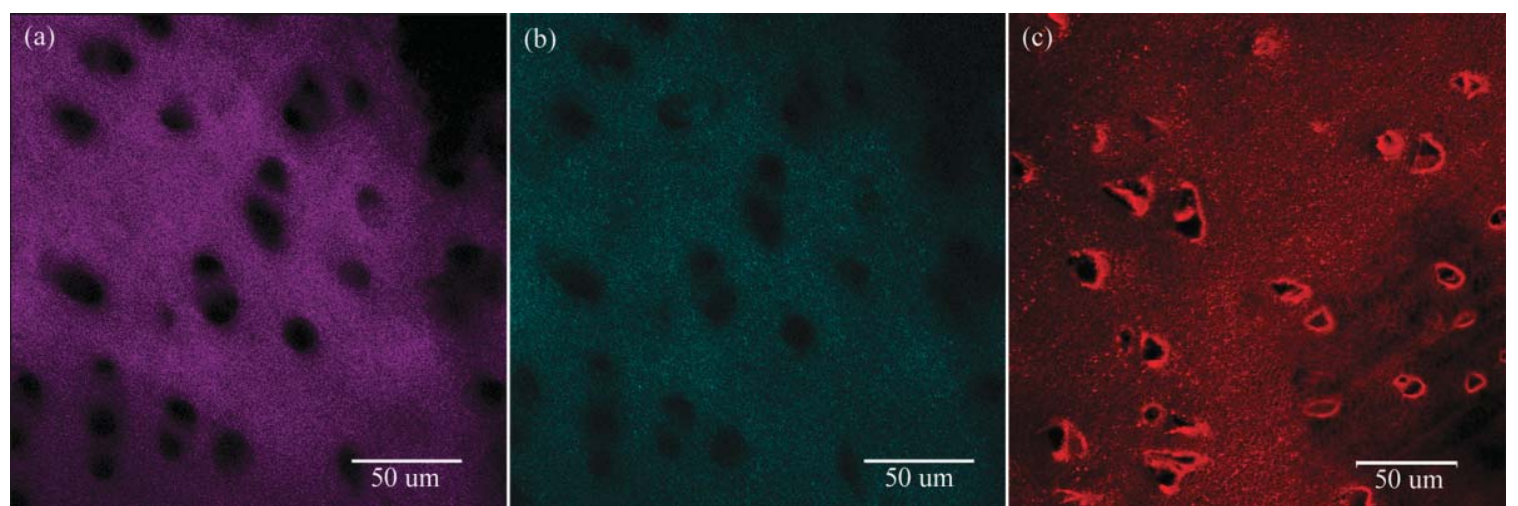

Fig. 4 Collagen in native pig cartilage. The SHG signal of collagen was much stronger in the backward mode (a) than in the forward one (b); (c) immunohistochemical staining of type II collagen using Cy3 detected by CLSM ( $\lambda_{\mathrm{exc}}=514 \mathrm{~nm}, \lambda_{\mathrm{em}}=555$ to $\left.600 \mathrm{~nm}\right)$. Maximum intensity projection, $20 \times$ objective, scale $=50 \mu \mathrm{m}$. 


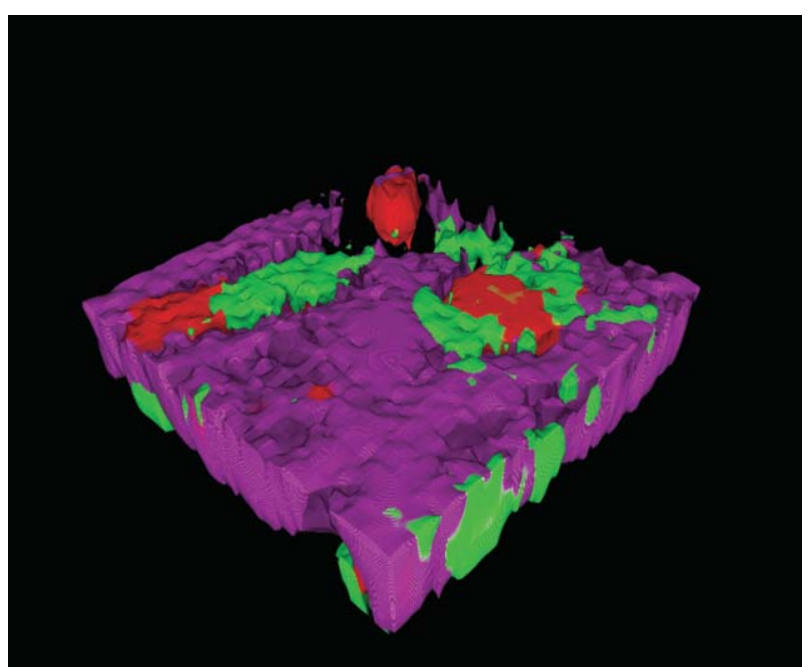

Video 1 A 3-D reconstruction of a PCL scaffold [displayed also in Fig. 2(e)]; collagen detected by SHG imaging in the backward mode (magenta), and by TPE excitation (green), cell nuclei stained by propidium iodide (red). Autofluorescence of the scaffold is also detected. (QuickTime, 10.4 MB).

[URL: http://dx.doi.org/10.1117/1.3509112.1]

images differed in the forward and backward modes. Bianchini and Diaspro ${ }^{9}$ and Cox et al. ${ }^{11}$ showed that the SHG signal in the forward mode is unable to pass through samples thicker than $500 \mu \mathrm{m}$. This finding indicates that the backward SHG detection is more efficient than the forward mode in thick samples, which is in accordance with our results obtained using 100 to $300-\mu \mathrm{m}$ thick samples.

Images obtained by employing either of two modes indicate a heterogeneous 3-D collagen distribution and are in agreement with collagen distribution determined by histochemical staining, although in short 5-day cell cultivation on scaffolds, the fluorescent collagen signal was found predominantly in the close

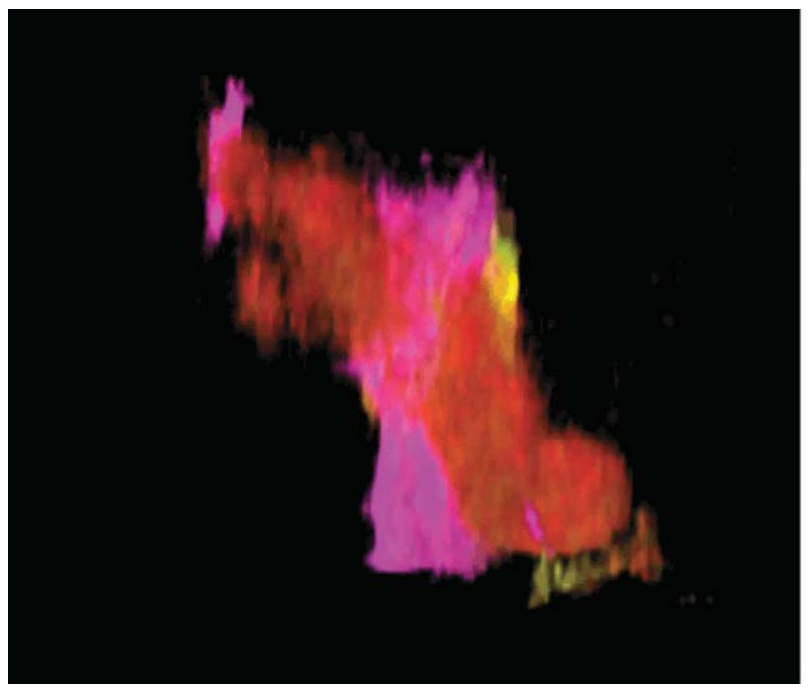

Video 2 A 3-D reconstruction of collagen on a gelatine scaffold in the backward mode (magenta) [displayed also in Fig. 3(b2)]; cell nuclei stained by propidium iodide (red), and autofluorescence of the scaffold (green) are also detected. (QuickTime, 8.7 MB).

[URL: http://dx.doi.org/10.1117/1.3509112.2] vicinity of the cells. This reflects the binding of the antibody to both procollagen and immature collagen (Fig. 2). On the contrary, the strong SHG signal was visible from well organized collagen in the extracellular matrix. This is in agreement with our previous observations in rabbits. ${ }^{20}$ Similarly, the sparse SHG signal in gelatine scaffold may be explained by the high amount of immature collagen in the sample.

Aside from collagen, natural and synthetic scaffolds can be visualized by CLSM, TPEM, and SHG imaging. ${ }^{18,21}$ Fluorescent amino acids (tryptophan, tyrosine and phenylalanine) in collagen, elastin, and other proteins; advanced glycation end products, flavin adenin dinucleotide; nicotinamide adenine dinucleotide; porphyrin; melanin; lipofuscin, and other natural fluorophores contribute to autofluorescence. ${ }^{22}$ The autofluorescence signal depends also on chemical composition of scaffolds; synthetic polyesters such as PCL contain the $\pi$-electrons necessary for fluorescence emission.

The type I and II collagen found in the scaffolds seeded with chondrocytes was produced by cells according to their state of differentiation, cell density, medium composition, and biomechanical stimulation. ${ }^{23,24}$ The SHG signal was sparsely distributed in the gelatine scaffolds, which was probably caused by a higher amount of immature collagen. A strong SHG signal was observed in a PCL scaffold [Fig. 2(d)]. The 3-D image reconstruction revealed a spatial distribution of mature collagen in the area with a high cell concentration [Figs. 2(e), 3(b2), and 3(c2) and Videos 1, 2, and 3]. The characterization of types of synthesized collagen using SHG in scaffolds will be the subject of future studies.

TPEM and SHG imaging have already been used for visualization of different tissues. ${ }^{25,26}$ In our experiments, CLSM, TPEM, and SHG imaging (forward and backward) was used for the visualization of in vitro prepared samples. Image analysis, stereological measurements, and 3-D reconstructions enabled us to quantify and visualize cell-collagen-scaffold systems, which had not yet been reported. We demonstrated the just mentioned

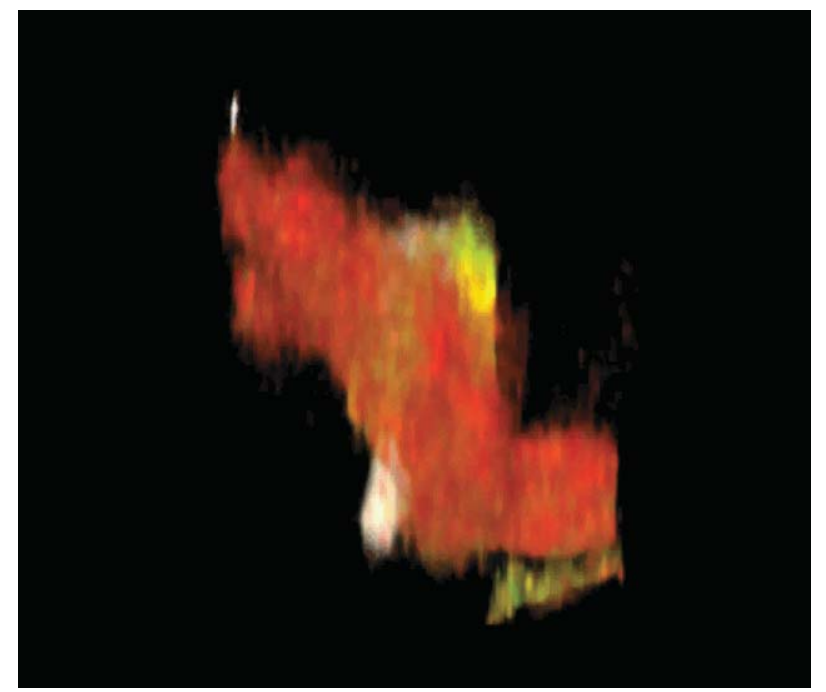

Video 3 A 3-D reconstruction of collagen on a gelatine scaffold in the forward mode (cyan) (displayed also in [Fig. 3(c2)]; cell nuclei stained by propidium iodide (red), and autofluorescence of the scaffold (green) are also detected. (QuickTime, 7.5 MB).

[URL: http://dx.doi.org/10.1117/1.3509112.3] 
techniques to be powerful tools for the analysis and visualization of collagen-containing scaffolds, with a broad application in cartilage tissue engineering.

\section{Summary}

SHG imaging enables us to perform 3-D reconstruction and visualization of cell-collagen-scaffold systems. Collagen volume can thus be reliably determined throughout the scaffold thickness. Moreover, a concurrent application of TPEM makes it possible to correlate scaffold and cell distribution in the samples.

\section{Acknowledgments}

The presented study was supported by the Academy of Sciences of the Czech Republic (research projects No. AV0Z50110509, AV0Z50390512, and AV0Z50390703), the Ministry of Education, Youth and Sports of the Czech Republic (research programs LC06063, MSM6840770012, NPV II 2B06130, and 1M0510), the Czech Science Foundation (Nos. GA102/08/0691, GA202/09/1151, and GAP304/10/1307), the EU project BIOSCENT (ID number 214539, and 7E09088), and the Grant Agency of the Academy of Sciences No. IAA500390702.

\section{References}

1. W. Denk, J. H. Strickler, and W. W. Webb, "Two-photon laser scanning fluorescence microscopy," Science 248, 73-76 (1990).

2. A. Diaspro, Ed., Confocal and Two-Photon Microscopy: Foundations, Applications, and Advances, Willey-Liss, New York (2002).

3. A. Diaspro, G. Chirico, and M. Collini, "Two-photon fluorescence excitation and related techniques in biological microscopy," Q. Rev. Biophys. 38, 97-166 (2005).

4. Y. Imanishi, K. H. Lodowski, and Y. Koutalos, "Two-photon microscopy: shedding light on the chemistry of vision," Biochemistry 46, 9674-9684 (2007).

5. P. J. Campagnola, A. C. Millard, M. Terasaki, P. E. Hoppe, C. J. Malone, and W. A. Mohler, "Three-dimensional high-resolution secondharmonic generation imaging of endogenous structural proteins in biological tissues," Biophys. J. 81, 493-508 (2002).

6. J. A. Palero, "Nonlinear spectral imaging of biological tissues," $\mathrm{PhD}$ Thesis, University of Utrecht, The Netherlands (2007)

7. F. Legare, C. Pfeffer, and B. R. Olsen, "The role of backscattering in SHG tissue imaging," Biophys. J. 93, 1312-1320 (2007).

8. R. M. Williams, W. R. Zipfel, and W. W. Webb, "Interpreting secondharmonic generation images of collagen I fibrils," Biophys. J. 88, 1377-1386 (2005).

9. P. Bianchini and A. Diaspro, "Three-dimensional (3D) backward and forward second harmonic generation (shg) microscopy of biological tissues," J. Biophoton. 1, 443-450 (2008).

10. O. Chernyavskiy, L. Vannucci, P. Bianchini, F. Difato, M. Saieh, and L. Kubínová, "Imaging of mouse experimental melanoma in vivo and ex vivo by combination of confocal and nonlinear microscopy," Microsc. Res. Tech. 72, 411-423 (2009).
11. G. Cox, P. Xu, C. Sheppard, and J. Ramshaw, "Characterization of the second harmonic signal from collagen," in Multiphoton Microscopy in the Biomedical Sciences III, A. Periasamy, P. T. C. So, Eds., Proc. SPIE 4963, 32-40 (2003).

12. K. G. Brockbank, W. R. MacLellan, J. Xie, S. F. Hamm-Alvarez, Z. Z. Chen, and K. Schenke-Layland, "Quantitative second harmonic generation imaging of cartilage damage," Cell Tissue Bank 9, 299-307 (2008).

13. J. A. Buckwalter and H. J. Mankin, "Articular cartilage: Tissue design and chondrocyte-matrix interactions," Instr. Course Lect. 47, 477-486 (1998).

14. B. Caterson, C. E. Hughes, B. Johnstone, and J. S. Mort. "Immunological markers of cartilage proteoglycan metabolism in animal and human osteoarthritis," in Articular Cartilage and Osteoarthritis, K. Kuettner, Ed., pp. 415-428, Raven Press, New York (1992).

15. R. A. Bank, M. Soudry, A. Maroudas, J. Mizrahi, and J. M. TeKoppele, "The increased swelling and instantaneous deformation of osteoarthritic cartilage is highly correlated with collagen degradation," Arthritis Rheum. 43, 2202-2210 (2000).

16. R. Dorotka, U. Windberger, K. Macfelda, U. Bindreiter, C. Toma, and S. Nehrer, "Repair of articular cartilage defects treated by microfracture and a three-dimensional collagen matrix," Biomaterials 26, 3617-3629 (2005).

17. S. Marlovits, P. Zeller, P. Singer, C. Resinger, and V. Vecsei, "Cartilage repair: generations of autologous chondrocyte transplantation," Eur. J. Radiol. 57, 24-31 (2006).

18. Y. Sun, H. Y. Tan, S. J. Lin, H. S. Lee, T. Y. Lin, S. H. Jee, T. H. Young, W. Lo, W. L. Chen, and C. Y. Dong, "Imaging tissue engineering scaffolds using multiphoton microscopy," Microsc. Res. Tech. 71, 140-145 (2008).

19. M. Čapek, P. Brůža, J., Janáček, P. Karen, L. Kubínová, and R. Vagnerová, "Volume reconstruction of large tissue specimens from serial physical sections using confocal microscopy and correction of cutting deformations by elastic registration," Microsc. Res. Tech. 72, 110-119 (2009).

20. E. Filová, F. Jelínek, M. Handl, A. Lytvynets, M. Rampichová, F. Varga, J. Činátl, T. Soukup, T. Trč, and E. Amler "Novel composite hyaluronan/type I collagen/fibrin scaffold enhances repair of osteochondral defect in rabbit knee," J. Biomed. Mater. Res. B Appl. Biomater. 87, 415-424 (2008).

21. Y. W. Wang, Q. Wu, J. Chen, and G. Q. Chen, "Evaluation of three-dimensional scaffolds made of blends of hydroxyapatite and poly(3-hydroxybutyrate-co-3-hydroxyhexanoate) for bone reconstruction," Biomaterials 26, 899-904 (2005).

22. D. Chorvat and A. Chorvatova, "Multi-wavelength fluorescence lifetime spectroscopy," Laser Phys. Lett. 6, 175-193 (2009).

23. S. E. Carver and C. A. Heath, "Influence of intermittent pressure, fluid flow, and mixing on the regenerative properties of articular chondrocytes," Biotechnol. Bioeng. 65, 274-281 (1999).

24. A. Nečas, L. Plánka, R. Srnec, M. Crha, J. Hlučilová, J. Klíma, D. Starý, L. Křen, E. Amler, L. Vojtová, J. Jančáŕ, and P. Gál, "Quality of newly formed cartilaginous tissue in defects of articular surface after transplantation of mesenchymal stem cells in a composite scaffold based on collagen I with chitosan micro- and nanofibres," Physiol. Res. 59, 605-614 (2010).

25. W. L. Chen, Y. Sun, W. Lo, H. Y. Tan, and C. Y. Dong, "Combination of multiphoton and reflective confocal imaging of cornea," Microsc. Res. Tech. 71, 83-85 (2008).

26. A. Zoumi, A. Yeh, and B. J. Tromberg, "Imaging cells and extracellular matrix in vivo by using second-harmonic generation and two-photon excited fluorescence," Proc. Natl. Acad. Sci. U.S.A. 99, 11014-11019 (2002). 